\title{
Resistência de Clones e Híbridos de Porta-Enxertos de Citros à Gomose de Tronco Causada por Phytophthora parasitica
}

\author{
Herculano P. Medina Filho ${ }^{1 *}$, Rita Bordignon${ }^{1}$, Walter J. Siqueira ${ }^{1}$, Eduardo Feichtenberger ${ }^{2}$, \\ Marli R. T. Carvalho ${ }^{1} \&$ Joaquim Teófilo Sobrinho ${ }^{1}$
}

${ }^{1}$ Instituto Agronômico, Cx. Postal 28, CEP 13001-970, Campinas, SP, e-mail: medina@iac.sp.gov.br; ${ }^{2}$ Unidade de Pesquisa e Desenvolvimento de Sorocaba, Rua Antonio G. Morgado, 340, CEP 18013-440, Sorocaba, SP

(Aceito para publicação em 30/07/2003)

Autor para correspondência: Herculano P. Medina Filho

MEDINA FILHO, H.P., BORDIGNON, R., SIQUEIRA, W.J., FEICHTENBERGER, E., CARVALHO, M.R.T. \& TEÓFILO SOBRINHO, J. Resistência de clones e híbridos de porta-enxertos de citros à gomose de tronco causada por Phytophthora parasitica. Fitopatologia Brasileira 28:534-540. 2003.

\section{RESUMO}

Sete grupos de híbridos entre porta-enxertos elite de citros (Citrus sp.) e seus genitores foram estudados quanto a reação à infecção de tronco por Phytophthora parasitica, em plantas adultas no campo. Inocularam-se artificialmente, em duas posições do tronco, 132 plantas nucelares dos genitores e 486 híbridos entre limão (Citrus limonia) Cravo 'Limeira' (C), Poncirus trifoliata 'Davis A' (T), tangerina (C.sunki) 'Sunki' (S) e laranja Azeda (C. aurantium) 'São Paulo' (A). A classificação do grau de resistência à gomose de tronco em plantas adultas no campo foi possível somente quando baseada na média do tamanho de lesões de mais de 30 plantas nucelares, devido às grandes variações observadas em plantas individuais do mesmo clone, impossibilitando a seleção precoce de híbridos baseada em valores de plantas individuais. O trifoliata e a laranja Azeda mostraram-se bastante resistentes, desenvolvendo, em geral, lesões de tamanho reduzido. A tangerina Sunki e o limão Cravo desenvolveram lesões maiores, embora a tangerina Sunki tenha mostrado uma tendência em desenvolver lesões maiores que às do limão Cravo. Híbridos de trifoliata apresentaram, no geral, lesões intermediárias. Os híbridos de Azeda apresentaram lesões de tamanhos bastante variáveis, porém a maioria, com lesões grandes. Da mesma forma comportaram-se os híbridos recíprocos entre Sunki e Cravo.

Palavras-chave adicionais: Citrus, melhoramento, portaenxertos, Phytophthora nicotianae, resistência genética.

\section{ABSTRACT}

Resistance of citrus rootstock clones and hybrids to trunk gummosis caused by Phytophthora parasitica

Seven groups of elite citrus (Citrus sp.) rootstock hybrids and their genitors were field tested for gummosis caused by Phytophthora parasitica. Two trunk inoculations were made in 132 nucellar clones of the genitors and in 486 hybrids between 'Limeira' rangpur lime (Citrus limonia) (C), 'Davis A' trifoliate orange (Poncirus trifoliata) (T), 'Sunki' mandarin (C. sunki) (S) and 'São Paulo' sour orange (C. aurantium) (A). The degree of resistance to gummosis could be estimated from inoculation of adult plants in the field only when it was based on the mean lesions of more than 30 nucellar plants. This was due to the great variations observed in single individuals of the same clone although the conditions were well controlled. For this reason, it was not possible to make an early selection of hybrids based on the mean lesion area developed in single individuals. Trifoliate and sour oranges were quite resistant, displaying small-size lesions. Sunki mandarin and rangpur lime developed larger lesions, although Sunki mandarin tended to develop larger lesions than rangpur lime. In general, trifoliate hybrids showed lesions of intermediate size. Sour orange hybrids developed lesions that were quite variable in size although most of them were large. This same behaviour was observed among reciprocal $\mathrm{SxC}$ hybrids.

\section{INTRODUÇÃO}

A incidência e a severidade das doenças em citros (Citrus spp.) causadas por Phytophthora parasitica Dastur $[P$. nicotianae Breda de Haan var. parasitica (Dastur) Waterhouse] e P. citrophthora (Sm. \& Sm.) Leonian têm aumentado muito nos últimos anos, constituindo-se em um dos principais problemas da cultura não só no Brasil como em vários outros países produtores (Carpenter \& Furr; 1962; Graham, 1990; 1995; Matheron et al., 1998). Nos Estados

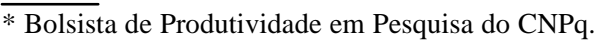

Unidos, na Flórida e no Texas, $P$. parasitica é a espécie mais comumente associada a essas doenças (Grimm \& Hutchison, 1973; Timmer \& Menge, 1988; Lutz et al., 1991; Zitko \& Timmer, 1994) chegando a ser responsável por $95 \%$ das infecções (Timmer, 1991).

No Estado de São Paulo, $P$. parasitica é também a espécie preponderantemente associada às perdas mais significativas provocadas por esses patógenos, tanto em viveiros, como em pomares comerciais (Feichtenberger, 2001). As doenças incitadas por Phytophthora spp. em citros são comumente referidas como gomoses que são conhecidas desde o século X, na Península Ibérica. A primeira grande epidemia 
de gomose dos citros ocorreu nas ilhas Açores, a partir de 1832, tendo sido observada desde 1845 em Portugal, com manifestações bastante severas. A partir desse foco, alastrou para a Espanha, tendo sido observada no sul da França em 1851, no norte da Itália em 1855, e, mais tarde na Grécia. Na América do Norte foi relatada em 1875 na Califórnia, tendo o primeiro surto importante da doença ocorrido na Flórida quatro anos mais tarde (Fawcett, 1936). Na América do Sul, o primeiro caso documentado ocorreu em 1709 em Lima, no Peru, e somente em 1917 foi detectada no Brasil (Alencar, 1941).

Algumas doenças têm limitado severamente a utilização de alguns porta-enxertos, não só no Brasil como em todos os países citrícolas do mundo. Nos Estados Unidos por exemplo, o limão rugoso (Citrus jambhiri Lush.) já foi o porta-enxerto utilizado em mais de $60 \%$ dos pomares embora, atualmente, seja raramente utilizado devido à sua suscetibilidade ao “declínio". O citrange Carrizo [Poncirus trifoliata (L.) Raf. x Citrus sinensis (L.) Osbeck], promissor substituto do limão rugoso mostra-se também suscetível ao "declínio". A utilização da laranja Azeda (Citrus aurantium L.), embora seja de grande aceitação, tem uso futuro incerto devido sua intolerância ao vírus da tristeza (Citrus tristeza virus, CTV). Em regiões infestadas com nematóides, poucas são as opções de portaenxertos resistentes. As laranjas-doce têm despertado grande interesse como porta-enxertos, por induzir altas produções e boa qualidade de frutos, mas seu uso é muito limitado devido a sua alta suscetibilidade à Phytophthora spp. (Carpenter\& Furr; 1962; Timmer, 1991; Graham, 1990; 1995; Matheronet al., 1998).

No Brasil, a laranja Azeda, considerada resistente à Phytophthora spp., era o porta-enxerto mais utilizado até a década de quarenta quando, devido a sua intolerância ao CTV, foi substituída pelo limão Cravo (Citrus limonia Osbeck), constituindo-se no principal porta-enxerto da citricultura brasileira. O limão Cravo é menos tolerante à infecção de Phytophthora spp. que a laranja Azeda, o que tem causado um aumento considerável na incidência e na severidade dos danos causados por esses patógenos.

São vários os danos causados pela infecção das espécies de Phytophthora, dependendo do órgão da planta infetado. É um problema sério em pomares adultos e viveiros causando "podridão de raízes". Os primeiros sintomas da "podridão do pé" ou gomose de Phytophthora spp. se manifestam com o escurecimento e a morte de pequenas regiões da casca do caule com exudação de goma no colo, principalmente em porta-enxertos suscetíveis. Os tecidos infetados da casca se rompem mostrando rachaduras e fendilhamentos longitudinais. Quando as lesões se desenvolvem muito, circundando grande parte do caule ou das raízes, a planta entra em rápido declínio, devido à destruição do floema, restringindo o fluxo de seiva elaborada da copa para o sistema radicular e provocando a morte da planta (Alencar, 1941; Rosseti, 1947; Timmer \& Menge, 1988; Feichtenberger, 1990; 2001).

Devido a importância econômica e ocorrência praticamente universal da gomose e da podridão das raízes, muitas investigações têm sido conduzidas no sentido de avaliar porta- enxertos de citros quanto à resistência a Phytophthora spp. O uso de porta-enxertos resistentes ou tolerantes constitui-se na principal forma de controle das doenças causadas por Phytophthora spp. A maioria das copas comerciais exibe relativa suscetibilidade às mesmas. $\mathrm{O}$ citrumelo Swingle e Citrus macrophylla Wester são classificados como tolerantes para as infecções de tronco e raízes. O limão Cravo é considerado como moderadamente suscetível, juntamente com a tangerina Sunki (Citrus sunki Hort. ex. Tan.), tangerina Cleópatra (C. reshni Hort. ex. Tan.), limão Volkameriano (Citrus volkameriana Ten. ePasq.) e citranges Troyer e Carrizo (Poncirus trifoliata $x$ C. sinensis). Outros porta-enxertos como o limão rugoso, pomelos (Citrus paradisi Macf.) e lima ácida [Citrus aurantifolia (Christm.) Swing.] apresentam uma alta suscetibilidade (Hutchison \& Grimm, 1973; Graham, 1990; 1995, Widmer et al.,1998).

Os primeiros testes de resistência à Phytophthora spp. em variedades de citros foram realizados por Fawcett (1923), o qual estabeleceu a natureza parasítica do patógeno. Klotz \& Fawcett (1930), utilizando o método de inoculação artificial do patógeno no tronco e avaliação da área da lesão resultante, investigaram a resistência de espécies e variedades de Citrus spp. e gêneros relacionados. Esse método foi subseqüentemente usado por outros pesquisadores (Fawcett \& Bitancourt, 1940; Rossetti, 1947).

Os mecanismos de resistência dos citros às infecções de Phythophthora, não estão ainda totalmente esclarecidos, mas é possível que vários mecanismos estejam envolvidos, devido às grandes diferenças observadas no tipo de resposta às infecções, em função do tipo e idade do tecido infetado. (Feichtenberger, 2001).

Em programas de melhoramento de porta-enxertos, onde se busca associar, em híbridos, diversas características de interesse agronômico como adaptabilidade edafoclimática e resistência a doenças, um dos primeiros critérios de seleção é o nível de resistência à Phytophthora spp. Neste trabalho, foram avaliados, quanto a reação à gomose de $P$. parasitica, quatro clones comerciais de porta-enxertos e 486 híbridos entre eles.

\section{MATERIAL E MÉTODOS}

\section{Material Vegetal}

No decorrer do programa de melhoramento de portaenxertos do Instituto Agronômico de Campinas, o Centro de Genética obteve um grande número de híbridos de portaenxertos, identificados e distinguidos das plantas nucelares por eletroforese de isoenzimas e marcadores morfológicos (Bordignon, 2000). Entre eles, aproximadamente 500 foram enxertados com copa de laranja 'Valência', transplantados para o campo em espaçamento de $8 \times 4 \mathrm{~m}$ no Centro Avançado de Pesquisa Tecnológica do Agronegócio de Citros Sylvio Moreira, em Cordeirópolis, e se encontram em fase de avaliação agronômica e tecnológica. Esses híbridos são provenientes de cruzamentos entre os clones $P$. trifoliata, $C$. aurantium, $C$. sunki, e C. limonia, indicados (Tabela 1). Devido à alta 


\section{H.P. Medina Filho et al.}

TABELA 1 - Número de porta-enxertos avaliados para resistência à infecção de tronco por Phytophthora parasitica em clones nucelares de Citrus spp., Poncirus trifoliata e híbridos entre eles

\begin{tabular}{|c|c|}
\hline Clonat/Híbridts & Nímerero de plantas \\
\hline \multicolumn{2}{|l|}{ C.Iontux } \\
\hline 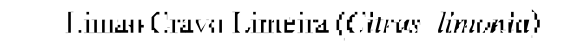 & 46 \\
\hline 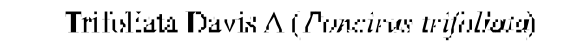 & 31 \\
\hline Tangerina Sunki ZUK (C. Sunki) & 46 \\
\hline Laranja Azeda Sāo l'anlo(C. anturltiun) & 8 \\
\hline T'ótal & 132 \\
\hline \multicolumn{2}{|l|}{ Híbridos } \\
\hline I.irmate Crava x lararij: A/eda & 41 \\
\hline Ianoerina Sunki x laran Azada & 90 \\
\hline lang̣erina Sunki $x$ linōo Crevo & 200 \\
\hline L.muâtr Crava x langèerina Suriki & 27 \\
\hline Tangerina Sunki $x$ P'oncuscs trifoliata & 56 \\
\hline Poneirus toffoliata $x$ tangerinat Stilki & 19 \\
\hline 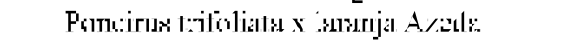 & 53 \\
\hline TitLal & 486 \\
\hline TOIAI. & 618 \\
\hline
\end{tabular}

heterozigozidade desses clones genitores, os híbridos entre eles representam, cada um, uma combinação genética diferente. Por outro lado, as plantas nucelares são geneticamente iguais, constituindo-se em clones maternos dos genitores utilizados nos cruzamentos.

\section{Obtenção de inóculos e inoculação das plantas}

Utilizou-se como fonte de inóculo o isolado de $P$. parasitica de número LRS 30/98 da Unidade de Pesquisa e Desenvolvimento de Sorocaba, da APTA Regional, SAA. A produção de propágulos do patógeno foi feita em meio de cultura de cenoura-agar (CA), descrito por Kaosiri et al. (1998). O micélio do patógeno foi obtido da margem de colônias de seis dias de idade, desenvolvidas a $24{ }^{\circ} \mathrm{C}$ no escuro, em meio de cultura CA cultivado em placas de Petri.

O método utilizado para a inoculação descrito por Rossetti (1947) se resume em: desinfecção com álcool da superfície da região do tronco a ser inoculada; incisão na casca com furador de rolhas, retirando-se um disco de $5 \mathrm{~mm}$ de diâmetro, expondo assim, a zona cambial; introdução de um disco de igual diâmetro de micélio de Phytophthora spp., proveniente da região de crescimento ativo do patógeno na placa de cultura; recolocação do mesmo disco da casca, anteriormente removido e proteção da inoculação com um pedaço de esparadrapo. Após a inoculação, protegeu-se essa região contra a dessecação, promovendo-se a uniformização do microambiente inoculado. Para tanto, enrolou-se firmemente no tronco, dois sacos de papel Kraft de $26,5 \mathrm{~cm}$ de largura de tal forma que, o perímetro do tronco ficasse protegido por oito a dez folhas desse papel que foram então grampeadas e assim mantidas até a avaliação dos sintomas.

Em cada planta foram feitas duas inoculações, sempre nas posições NE e SO, na altura mediana do porta-enxerto, a, aproximadamente, $10 \mathrm{~cm}$ do solo. No período compreendido entre a inoculação das plantas e a avaliação das lesões desenvolvidas, a temperatura média ambiente, em abrigo meteorológico próximo ao campo, variou de $18,4{ }^{\circ} \mathrm{C}$ a $31,4^{\circ} \mathrm{Ce}$ a umidade relativa média diária de $65,8 \%$ a $66,7 \%$. As lesões decorrentes foram avaliadas após 22 dias da inoculação, medindo-se o comprimento (c) e largura (1) da área lesionada observada após a remoção da casca do tronco próxima a inoculação, expondo-se totalmente a lesão desenvolvida. Para o cálculo da área lesionada, considerou-se a área da elipse ( $\pi$ $\mathrm{x} \mathrm{c} / 2 \times 1 / 2$ ) por representar melhor que a retangular, a forma irregular das lesões.

A fim de investigar possíveis correlações entre a área lesionada por P. parasitica, o estado vegetativo e a produção de frutos pendente na planta, considerou-se o vigor das plantas estimado a partir da expressão: [diâmetro médio da copa em cm + altura da planta em $\mathrm{cm}+(10 \mathrm{x}$ diâmetro do tronco em cm)] /100, de acordo com Bordignon (2000).

As médias dos clones e híbridos foram comparados pelo teste de Tukey ao nível de 5\% de significância com auxílio dos programas EXCEL e ORIGIN. Análises de Componentes Principais (ACP) foram realizadas com o programa STATITCF, considerando-se área da lesão associada a outros caracteres de vigor obtidos para essas mesmas plantas por Bordignon (2000), com os eixos principais calculados a partir do perímetro do tronco (PT), altura da planta (ALT), diâmetro da copa (DCP), índice geral de vigor (IV) e produção pendente (PD).

Paralelamente a este estudo foram feitas duas inoculações, de modo semelhante, em 25 plantas adultas e vigorosas de pé-franco de laranja Azeda, com objetivo de se estudar melhor o comportamento deste clone, uma vez que as plantas no campo de seleção estavam enxertadas com laranja Valência e, devido à sua intolerância ao CTV, encontravam-se pequenas e com reduzido vigor.

\section{RESULTADOS E DISCUSSÃO}

A avaliação da resistência à infecção de tronco por $P$. parasitica, através da área da lesão provocada pelo patógeno após inoculação dupla (NEe SO) no tronco das plantas, mostrou, pelo teste $t$, não haver diferença estatística significativa entre as posições de inoculação no desenvolvimento das lesões. Nas análises e considerações subseqüentes foi utilizada então a média das duas lesões desenvolvidas em cada planta.

As médias e a variação observada nas áreas das lesões desenvolvidas nos clones genitores e nos híbridos correspondentes foram devidamente registradas (Tabela 2 e Figura 1). Com respeito aos clones, os resultados obtidos mostraram que no trifoliata e na laranja Azeda desenvolveram-se lesões significativamente menores que na tangerina Sunki e no limão Cravo. A Sunki embora não diferindo estatisticamente do limão Cravo mostrou, entretanto, uma tendência para desenvolver lesões maiores (Figura 1), com médias e amplitudes de variação maiores que as do limão Cravo. De outra forma, essa mesma tendência pode ser verificada ao se analisar os valores limites dos terceiros quartis de ambos. Enquanto que no limão Cravo esse valor é de $5,5 \mathrm{~cm}^{2}$, na tangerina Sunki ele se eleva a 6,5 
TABELA 2 - Área da lesão média nos troncos de porta-enxertos de clones e híbridos de Citrus spp. e Poncirus trifoliata inoculados com micélio de Phytophthora parasitica

\begin{tabular}{|c|c|}
\hline Clıne/Ilílbrido & I.esàno $\left(\mathrm{smol}^{2}\right)^{*}$ \\
\hline \multicolumn{2}{|l|}{ C.Inme } \\
\hline Taugerina Sunki & $5,17 a$ \\
\hline Timân Cravo & $4,58, a$ \\
\hline Trï̈̈llial!ı Davis A & 2,0512 \\
\hline [ӓrranja A/edir (Cordeiro]nolis) & $0.87 a$ \\
\hline [.aranja Aneda (Nuclea lixp. Camp.] & $2,57 b$ \\
\hline \multicolumn{2}{|l|}{ Hílurido } \\
\hline Cravo $\times$ Sunki & $5,81 a$ \\
\hline Sunki x Cravo & $4,67 a$ \\
\hline Cravo $x \wedge z e d a$ & $4,13 a$ \\
\hline Sunki x Azeda & $4,10: x$ \\
\hline Ir:follinta x Azoda & 2966 \\
\hline Sutıki $x$ Trï̈ulisaly & $2,46 b$ \\
\hline Tr:bilialia $\times$ Strnki & 2,36 b \\
\hline
\end{tabular}

* Médias seguidas pela mesma letra não diferem significativamente pelo teste de Tukey a $5 \%$.

$\mathrm{cm}^{2}$. A mesma tendência é mostrada pela amplitude de variação nesse terceiro quartil que é, respectivamente, 9,5 e $13,5 \mathrm{~cm}^{2}$. Essas diferenças embora não sejam muito grandes vêm, entretanto, corroborar o que se conhece na citricultura comercial na qual viveiristas, produtores e extensionistas afirmam, pela prática com a cultura, que a tangerina Sunki é mais suscetível à gomose de Phytophthora spp. que o limão Cravo. De acordo com Feichtenberguer $(1990 ; 2001)$ ambos são moderadamente suscetíveis. A laranja Azeda apresentou a menor área de lesão e a menor amplitude de variação $\left(2 \mathrm{~cm}^{2}\right)$. Cabe ressaltar, entretanto, que as plantas enxertadas nesse clone apresentamse com crescimento extremamente reduzido devido à intolerância desse clone ao CTV multiplicado amplamente pelas copas de laranja 'Valência' neles enxertadas (Bordignon, 2000). Essa redução do tamanho das lesões por interferência de outras doenças e pelo estado vegetativo das plantas, também foi observada por Rossetti \& Bitancourt (1951); Rossetti \& Musumeci (1962); Carpenter \& Furr (1962); Rossetti (1969). O comportamento desse clone em boas condições de vigor, sem manifestações severas de tristeza, pode ser aferido pelos resultados das inoculações nas 25 plantas adultas de pé franco nas quais foram observadas lesões maiores (Tabela 2).

É notório entre os clones (Figura 1), a superioridade do trifoliata a qual se expressa por uma reduzida área de lesões e por uma reduzida amplitude de variação. Neste clone, o limite do terceiro quartil é $2,6 \mathrm{~cm}^{2}$ e a lesão máxima, $3,6 \mathrm{~cm}^{2}$. Os trifoliatas são conhecidos como porta-enxertos resistentes à gomose de Phytophthora spp. e os resultados aqui obtidos confirmam plenamente os resultados obtidos anteriormente com esse porta-enxerto.

Os híbridos entre tangerina Sunki (S), limão Cravo (C) e laranja Azeda (A) apresentaram as maiores médias de área de lesão e também uma grande amplitude de variação (Figura 2), exceto aqueles em que um dos genitores é o trifoliata ( $\mathrm{T}$ ). Nesses últimos casos, não somente são menores as médias, como também as amplitudes de variação dos terceiros quartis.

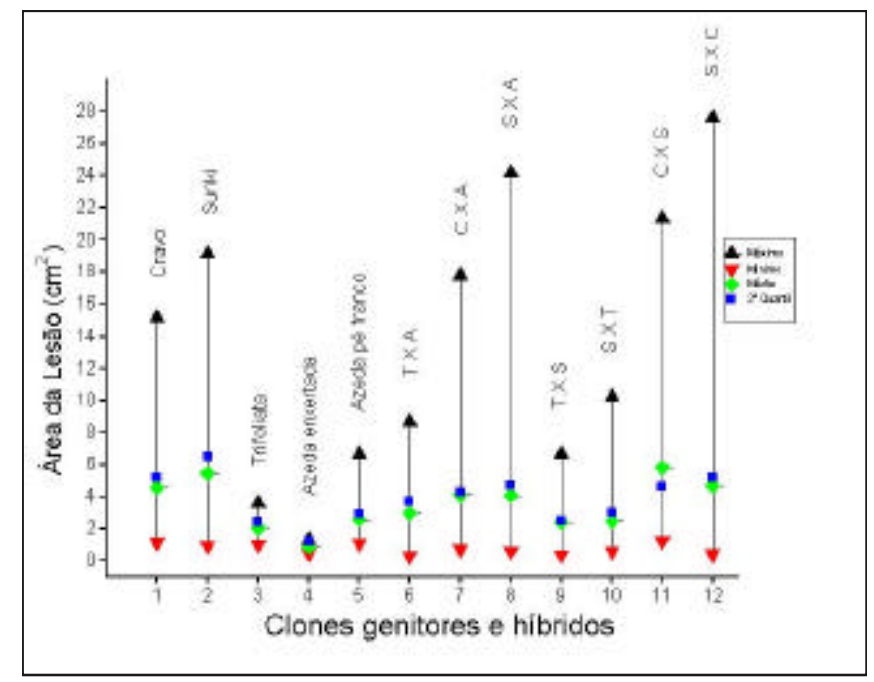

FIG. 1 -Estatísticas descritivas (valor máximo, mínimo, média e terceiro quartil) das áreas das lesões desenvolvidas após inoculação de Phytophthora parasitica no tronco dos genitores limão (Citrus limonia) Cravo (C), tangerina (C. sunki) Sunki (S), laranja Azeda (C. aurantium) (A), Poncirus trifoliata (T) e de seus híbridos

Consideráveis variações nos tamanhos das lesões foram também observadas por outros pesquisadores (Carpenter \& Furr, 1962; Matheronet al., 1998), razão pela qual sugerem que na análise das lesões sejam consideradas as condições fisiológicas das plantas, como o vigor e a interferência de outras doenças. No presente trabalho, como está envolvido grande número de híbridos, com combinações genéticas diversas, é possível considerar interações genéticoambiente com respeito à reação de resistência a Phytophthora spp. Esses híbridos, conforme relatado por Bordignon (2000), apresentam uma enorme variação quanto ao vigor, produção, precocidade de produção, produtividade por $\mathrm{m}^{2}$, enfolhamento, tamanho dofruto, brix e acidez do suco entre outras características, além de uma reação variável de tolerância à tristeza, principalmente entre os híbridos de laranja Azeda e trifoliata. No geral, os híbridos apresentaram uma amplitude de variação bem maior que os genitores. Os híbridos de tangerina Sunki e limão Cravo com trifoliata têm, em média, lesões muito menores, com menores variações que as observadas em limão Cravo x tangerina Sunki ou tangerina Sunki x limão Cravo sendo, portanto, mais provável encontrarem-se híbridos mais resistentes que Sunki ou que Cravo entre seus híbridos com trifoliata. De fato, os híbridos de trifoliata tiveram lesões bem inferiores aos híbridos que não são provenientes de hibridações deste clone, evidenciando que o trifoliata transmite à maioria de seus híbridos pelo menos um considerável grau de resistência à gomose de tronco por $P$. parasitica.

Os híbridos limão Cravo x laranja Azeda e tangerina Sunki x laranja Azeda desenvolveram lesões grandes comparativamente ao trifoliata ou à própria laranja Azeda. Isso parece indicar que a resistência da laranja Azeda parece não ser expressa em seus híbridos. As lesões menores dos híbridos 

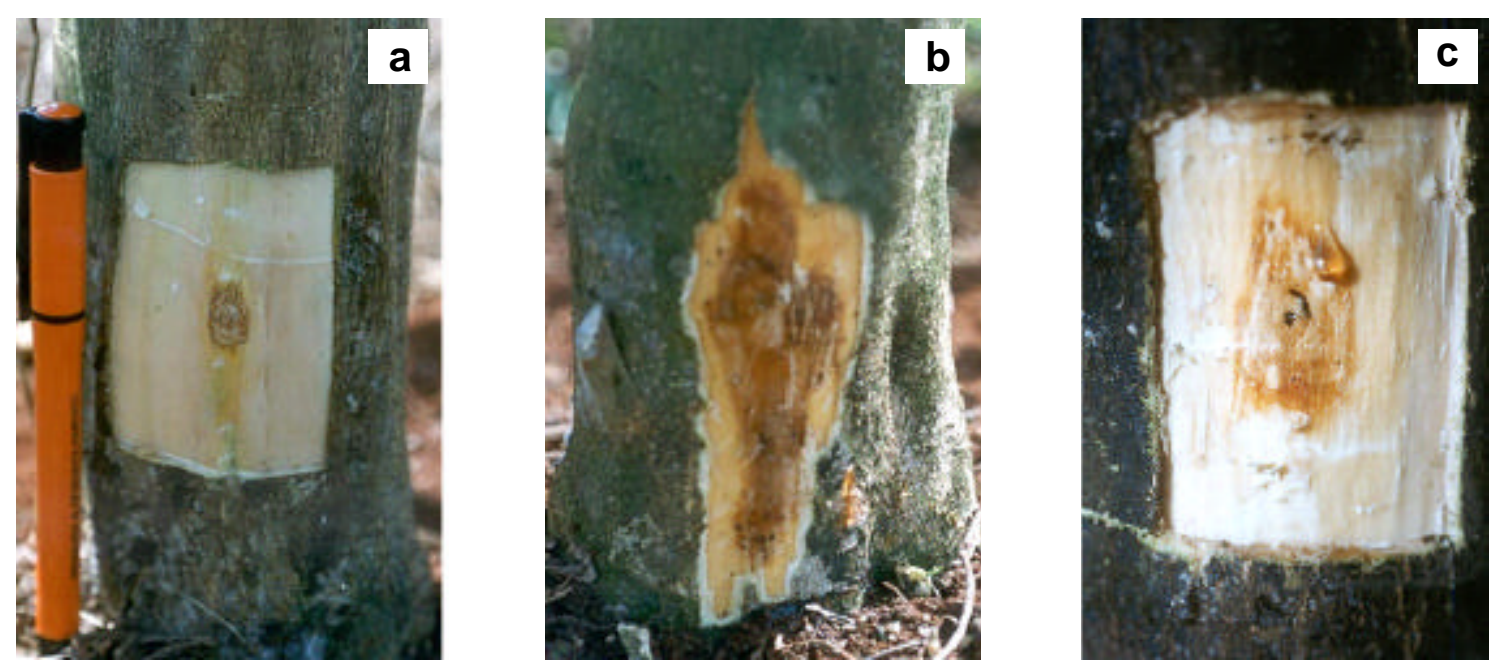

FIG. 2 - Variações no tamanho das lesões de Phytophthora parasitica observadas em troncos de híbridos de porta-enxertos de citros (Citrus spp.).

trifoliata x laranja Azeda são, por esta razão, devidas ao trifoliata, da mesma forma que os híbridos de Trifoliata x tangerina Sunki e seus recíprocos tangerina Sunki x trifoliata. Os híbridos de laranja Azeda (CxA, SxA, TxA) possuem uma tendência, evidenciada pela amplitude do terceiro quartil, em produzir híbridos com lesões maiores (Figura 1).

A análise de componentes principais mostrou que, apenas a v ariável produção pendente (PD) não apresentou correlação com a área da lesão (AL) desenvolvida na planta. Por esse motivo a PD não foi considerada nas análises subsequentes. De maneira geral, a variabilidade (Figura 3; Figura 4; Figura 5) foi superior a $90 \%$, com exceção dos híbridos de S x C e recíprocos que apresentaram respectivamente $77 \%$ e $76,5 \%$. O eixo 1, explicado pelas variáveis PT, ALP, DCP e IV poderia ser denominado eixo do vigor e o eixo 2, explicado pela variável AL, seria o eixo da reação a Phytophthora spp. expresso pela característica área da lesão. Assim, no eixo 1 a direção do vetor IV indica o grau crescente de vigor das plantas analisadas e, no eixo 2, quanto mais abaixo, menor a área da lesão desenvolvida e maior a possibilidade de um nível superior de resistência.

Para os clones, a dispersão dos indivíduos sugere a existência de três grupos distintos (Figura 3), separados pelo vigor vegetativo das plantas e pelo nível de resistência, representados nos eixos 1 e 2 , respectivamente. O primeiro grupo, representado pela laranja Azeda, apresenta menor vigor vegetativo e pequena área de lesão. Um segundo grupo representado pelos indivíduos de trifoliata apresentam um nível de vigor intermediário e de resistência ligeiramente superior ao da Azeda. O terceiro grupo é composto da tangerina Sunki e do limão Cravo, com plantas mais vigorosas que aquelas enxertadas em trifoliata e azeda, apresentando, porém, uma maior variabilidade na área das lesões. Nesses grupos, ocorre um razoável número de indivíduos com lesões grandes, situados, no gráfico, na parte superior do eixo 2 , sendo mais suscetíveis a gomose quando comparados com trifoliata ou com a laranja Azeda. A classificação geral dos indivíduos nesses grupos sugeridos pela ACP e realizada através de uma Análise Fatorial Discriminante (AFD) revelou que 93,1\% das plantas foram bem classificadas nos três grupos distintos.

Uma análise dos híbridos, relacionando a área de lesão e o vigor vegetativo das plantas, foi realizada tomando-se o trifoliata como padrão de resistência à gomose de Phytophthora spp. De acordo com os resultados obtidos para as diferentes combinações híbridas (Figuras 4 e 5), e da mesma forma que na análise realizada nos clones, os híbridos foram também separados em função do vigor vegetativo e da área da lesão desenvolvidas nas plantas, explicados, respectivamente, pelos eixos 1 e 2. Em todas as combinações híbridas observou-se uma grande proporção de plantas que apresentaram áreas de lesão menor ou igual à máxima observada no trifoliata. Especificamente, a porcentagem desses híbridos foram $84 \%$ nos cruzamentos entre tangerina Sunki e trifoliata, $77 \%$ entre trifoliata e laranja Azeda, 66\% entre limão Cravo e laranja Azeda, 63\% entre tangerina Sunki e laranja Azeda e 60\% entre limão Cravo e tangerina Sunki. Esses dados corroboram plenamente

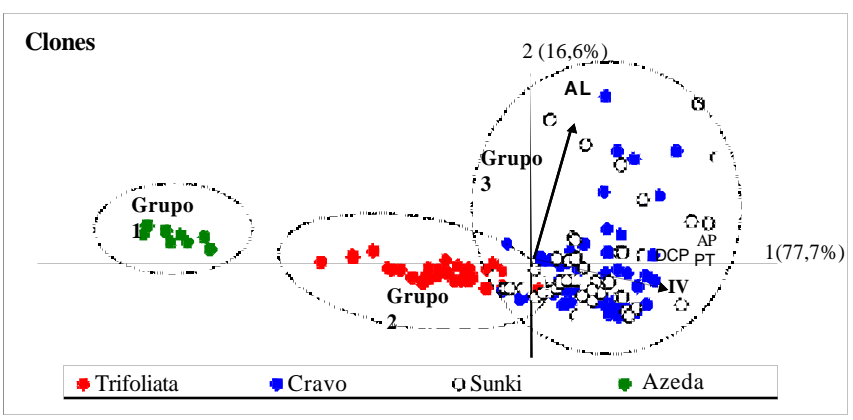

FIG. 3 - Análise de Componentes Principais. Associação entre as variáveis área de lesão (AL); perímetro do tronco (PT); diâmetro da copa (DCP); índice de vigor (IV). Representação no plano 1/2 dos indivíduos pertencentes aos clones trifoliata, limão (Citrus limonia) Cravo, tangerina (C. sunki) Sunki e laranja Azeda (C. aurantium). 
Resistência de clones e híbridos de porta-enxertos de citros...
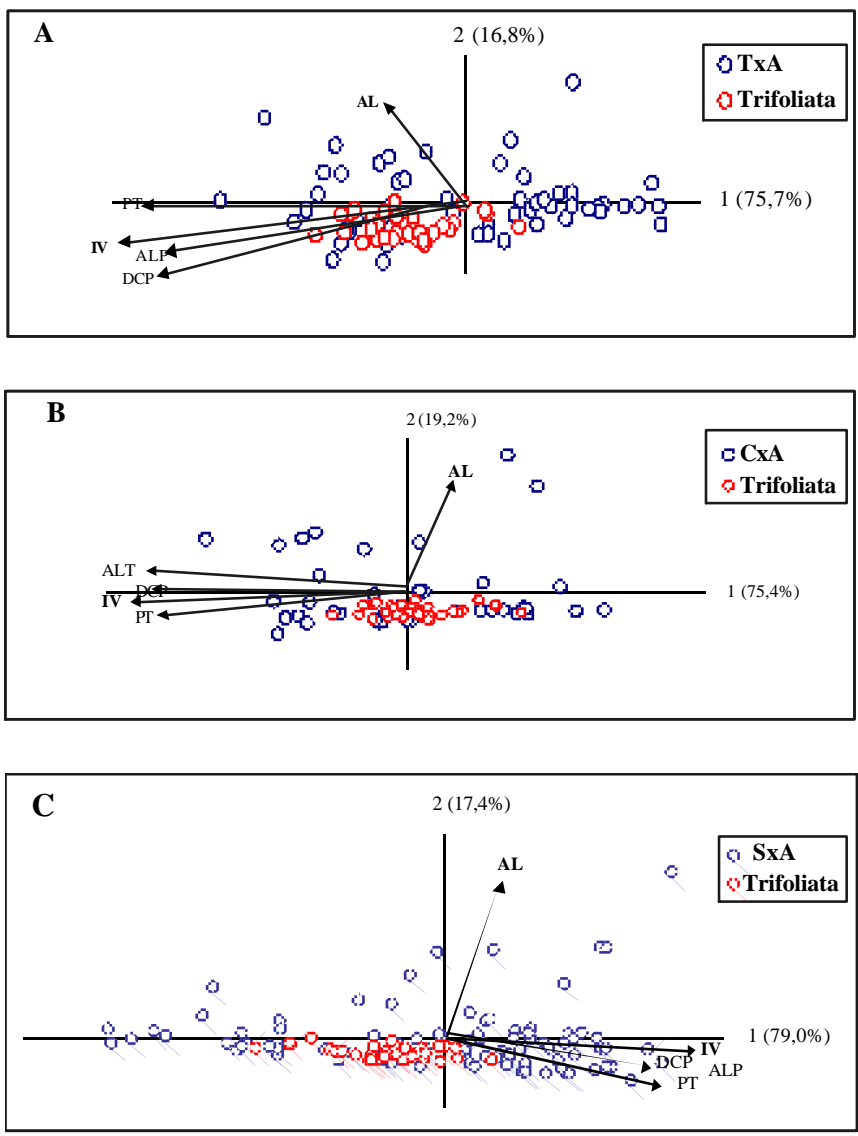

FIG. 4 - Análise de Componentes Principais. Associação entre as variáveis área de lesão (AL), perímetro do tronco (PT), diâmetro da copa (DCP), altura da planta (AL) e índice de vigor (IV). Representação no plano 1/2 dos indivíduos pertencentes aos híbridos: A) trifoliata (T) x laranja Azeda (Citrus aurantium) (A); B) limão (C. limonia) Cravo (C) x laranja Azeda; C) tangerina (C. sunki) Sunki (S) x laranja Azeda.

as considerações anteriores baseadas nas análises dos limites dos terceiros quartis e nas amplitudes de variação.

Os híbridos dos cruzamentos recíprocos entre Sunki e Cravo são muito mais vigorosos que o trifoliata. Nas demais combinações híbridas, observou-se existir sempre entre eles, uma certa proporção de plantas com baixo vigor. Neste caso, o menor vigor é devido à herança dessa característica do trifoliata, o qual induz à copa de 'Valência' um menor porte devido a uma certa incompatibilidade copa/porta-enxerto. Uma segunda possibilidade seria a segregação para intolerância à tristeza, com os híbridos intolerantes apresentando um vigor muito reduzido. Entre os híbridos S x A e S x C existe razoável número de indivíduos vigorosos e com reduzida área de lesão de gomose. Entre tais híbridos poderiam existir alguns portaenxertos potencialmente promissores para essas duas características (Bordignon, 2000). Entretanto, com base nos resultados do presente trabalho, a seleção, mesmo visando identificar grandes diferenças de tolerância da magnitude entre o trifoliata e a tangerina Sunki ou o limão Cravo, somente seria segura se baseada em avaliações de progênies constituídas de mais de
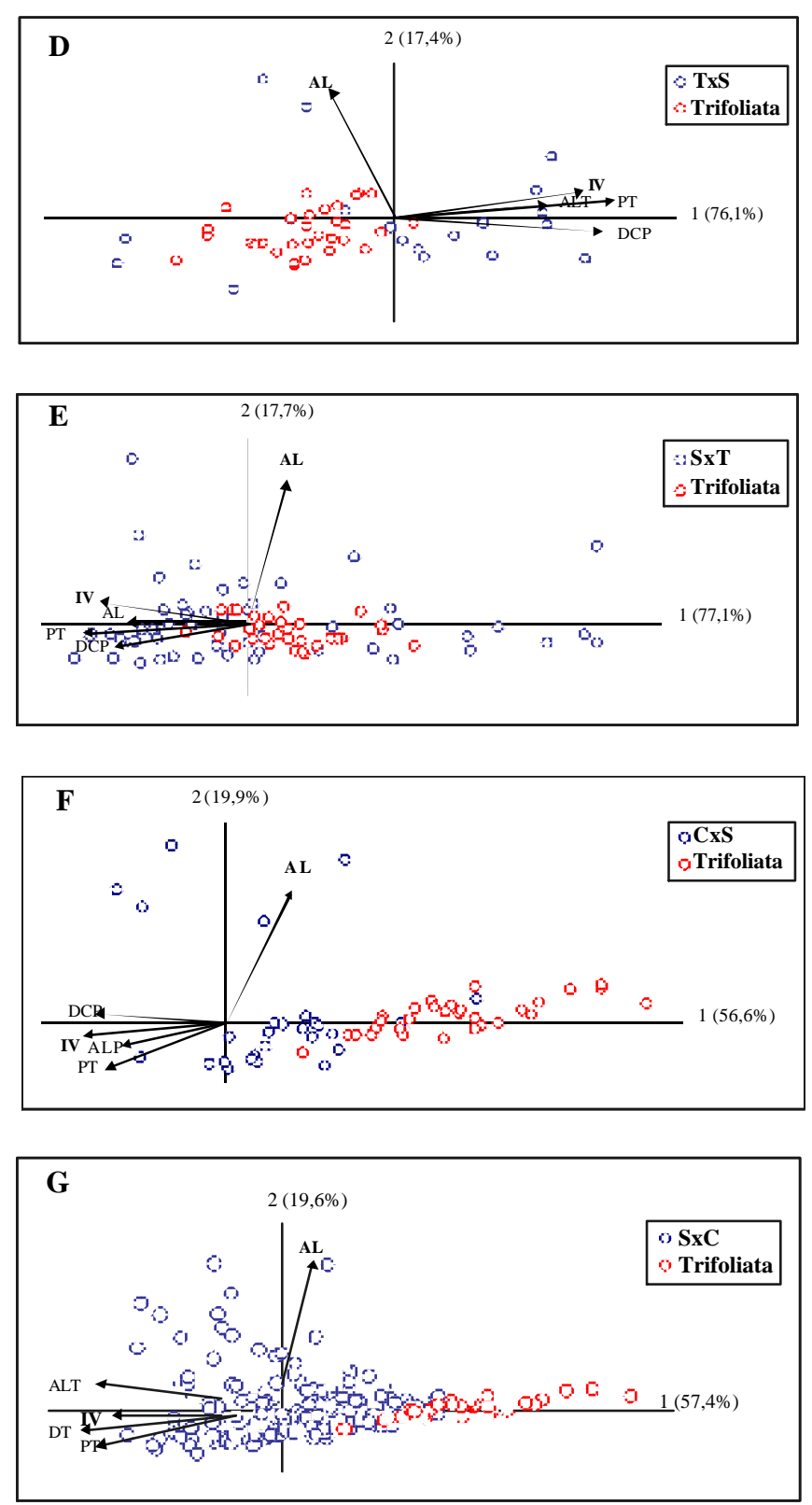

FIG. 5 - Análise de Componentes Principais. Associação entre as variáveis área de lesão (AL), perímetro do tronco (PT), diâmetro da copa (DCP), altura da planta (ALT) e índice de vigor (IV). Representação no plano 1/2 dos indivíduos pertencentes aos híbridos: D) Trifoliata (T) x tangerina (Citrus sunki) Sunki (S); E) tangerina Sunki (C. sunki) $\mathrm{x}$ trifoliata; $\mathrm{F}$ ) limão (C. limonia) Cravo $\mathrm{x}$ tangerina Sunki e G) tangerina Sunki x limão Cravo.

30 plantas nucelares desses híbridos devido as variações observadas nas reações de plantas individuais em condições de campo.

\section{AGRADECIMENTOS}

A J. Pompeu Junior pelas sugestões ao trabalho, a R. M. L. Ballvé pela ajuda nas avaliações das lesões e a $O$. Guerreiro Filho pela ajuda nas ACP e AFD. 


\section{H.P. Medina Filho et al.}

\section{REFERÊNCIAS BIBLIOGRÁFICAS}

ALENCAR, J. Podridão do pé dos citros. Boletim ñ 6, Escola Superior de Agricultura de Minas Gerais, Viçosa. 1941.

BORDIGNON, R. Características agronômicas e tolerância à tristeza de híbridos entre porta-enxertos elite de citros . (Tese de Doutorado). Campinas. Universidade Estadual de Campinas. 2000.

CARPENTER, J.B \& FURR, J.R. Evaluation of tolerance to root rot caused by Phytophthora parasitica in seedlings of Citrus and related genera. Phytopathology 52:1277-1285. 1962.

FAWCETT. H.S. Gummosis of citrus. Journal of Agricultural Research 24:191-236. 1923.

FAWCETT, H.S. Citrus disease and their control. $2^{\text {nd }}$ ed. New York. Mcgraw Hill Co. 1936.

FAWCETT, H.S. \& BITANCOURT, A.A. Occurence, pathogenicity and temperature relations of Phytophthora species on citrus in Brazil and other South American countries. Arquivos do Instituto Biológico 11:107-121. 1940.

FEICHTENBERGER, E. Control of Phytophthora gummosis of citrus with systemic fungicides in Brazil. EPPO Bulletin 20:139148. 1990.

FEICHTENBERGER, E. Doenças incitadas por Phytophthora em citros. In: Luz, E. D. M. N.; Santos, A. F.; Matsuoka, K.; Bezerra, J.L. (Eds). Doenças causadas por Phytophthora no Brasil. Campinas. Livraria e Editora Rural Ltda. 2001. pp.283-342.

GRAHAM, J.H. Evaluation of tolerance of citrus rootstocks to Phytophthora root rot in chlamydospore infested soil. Plant Disease 74:743-746. 1990.

GRAHAM, J.H. Root regeneration and tolerance of citrus rootstocks to root rot caused by Phytophthora nicotianae. Phytopathology 85:111-117. 1995.

GRIMM, G.R. \& HUTCHISON, D.J. A procedure for evauating resistance of citrus seedlings to Phytophthora parasitica. Plant Disease Reporter 57:669-672. 1973.

HUTCHISON, D.J. \& GRIMM, G.R. Citrus clone resistance to Phytophthora parasitica. Screening results. Proceedings of Florida State Horticultural Society 86:88-91. 1973.

KAOSIRI, T.; ZENTMYER, G.A. \& ERWIN, D.C. Stalk lengt h as a taxonomic criterion for Phytophthora palmivora isolates from cacao. Canadian Journal of Botany 56:1730-1738. 1998.

KLOTZ, L.J. \& FAWCETT, H.T.S. The relative resistance of varieties and species of citrus to Phythiacystis gummosis and other bark diseases. Journal of Agricultural Research 41:415-425. 1930. LUTZ, A.L. \& MENGE, J.A. Population fluctuations and the numbers and types of propagules of Phytophthora parasitica that occur in irrigated citrus groves. Plant Disease. 75:173-179. 1991.

MATHERON, M.E.; WRIGHT, G.C. \& PORCHAS, M. Resistance to Phytophthora citrophthora and P. parasitica and nursery characteristics of several citrus rootstocks. Plant Disease 82:1217-1225. 1998.

ROSSETTI, V. Estudos sobre "gomose de Phytophthora" dos citros I. Suscetibilidade de diversas espécies cítricas à algumas espécies de Phytophthora. Arquivos do Instituto Biológico 18:97124. 1947.

ROSSETTI, V. Studies on Phytophthora species on Citrus in Brasil. Proceedings, First International Citrus Symposium, Riverside.1969. pp.1211-1216.

ROSSETTI, V. \& BITANCOURT, A.A. Estudos sobre a "gomose de Phytophthora" dos citros II. Influência do estado de vegetação do hospedeiro nas lesões experimentais. Arquivos do Instituto Biológico 20:73-94. 1951.

ROSSETTI, V. \& MUSUMECI, M.R. Influência da variedade da copa de plantas cítricas sobre o comportamento do porta-enxerto com relação à gomose de Phytophthora. Ciência e Cultura 14:182183. 1962.

TIMMER, P. Identification and control of Phytophthora diseases. Citrus Industry 4:73-75. 1991.

TIMMER, L.W. \& MENGE, J.A. Phytophthora-induced diseases. In: Whiteside, J.O., Garnsey, S.M. \& Timmer, L.W. (Eds). Compendium of Citrus diseases. Saint Paul. American Phytopathological Society Press. 1988. pp.22-24.

WIDMER, T.L.; GRAHAM, J.H. \& MITCHELL, D.J. Histological comparasion of fibrous root infection of disease- tolerant and susceptible citrus hosts by Phytophthora nicotianae and $P$. palmivora. Phytopathology 88:389-395. 1998.

ZITKO, S.E. \& TIMMER, L.W. Competitive parasitic abilities of Phytophthora parasitica and P. palmivora on fibrous roots of citrus. Phytopathology 84:1000-1004. 1994. 